\title{
Microstructural Analysis of a Silver-Plated Trombone
}

\author{
Christopher Marvel $\cdot$ Will Lenthe $\cdot$ John Logan
}

Received: 9 February 2012/ Accepted: 9 February 2012/Published online: 8 March 2012

(C) Springer Science+Business Media, LLC and ASM International 2012

\section{Introduction}

This investigation focused on utilizing metallographic procedures to identify the processing techniques of a joint on a silver-plated brass trombone manufactured ca. 1910, illustrated in Fig. 1. Specifically, the joint between the mouthpiece receiver and a brace was examined, Fig. 2.

\section{Experimental Procedure}

The joint was sectioned, mounted, and polished with standard metallographic procedures. Due to the fact that several different materials were used in this joint, it was not possible to reveal all microstructures simultaneously with a single etchant. The metallographic sample, shown in Fig. 3, prepared from the location shown in Fig. 2, was initially etched with a potassium dichromate solution: $2 \mathrm{~g}$ $\mathrm{K}_{2} \mathrm{Cr}_{2} \mathrm{O}_{7}, 8 \mathrm{~mL} \mathrm{H}_{2} \mathrm{SO}_{4}, 4$ drops $\mathrm{HCl}, 125 \mathrm{~mL} \mathrm{H} \mathrm{H}_{2} \mathrm{O}$. The sample was viewed under crossed polars to reveal the brass microstructure (Regions 1, 3, and 5 in Fig. 4). The lead-tin solder of the brace joint was severely attacked by the potassium dichromate. The sample was re-polished and imaged in the as-polished condition, Fig. 5.

Editor's Note The 2011 Jacquet-Lucas Award for Excellence in Metallography was awarded to a team of undergraduate materials science students from Lehigh University. The authors, pictured here with their winning entry, analyzed the microstructures found in a brass trombone. This short communication from the authors highlights their major findings.

C. Marvel $(\bowtie) \cdot$ W. Lenthe $\cdot$ J. Logan

Materials Science and Engineering, Lehigh University,

5 East Packer Ave., Bethlehem, PA 18015, USA

e-mail: cjm312@lehigh.edu

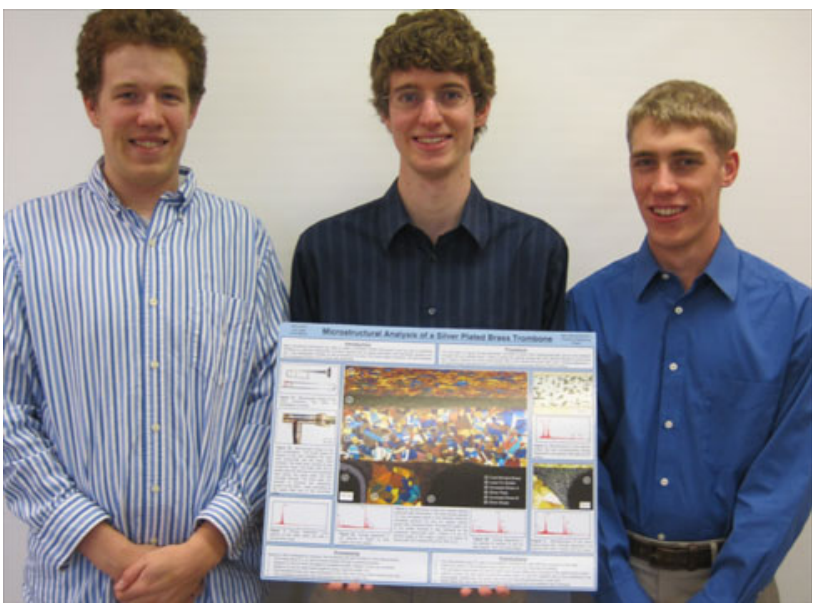

(From left) Chris Marvel, John Logan, and Will Lenthe show their winning International Metallographic Contest poster

To reveal the microstructure of the silver braze (Region 6, Fig. 4), the specimen was etched with a modified Stewart's reagent: A solution of $50 \mathrm{~mL} 3 \%$ hydrogen peroxide and $50 \mathrm{~mL}$ ammonium hydroxide diluted with $25 \mathrm{~mL}$ of water.

The as-prepared samples were examined under a light microscope (LM), and a scanning electron microscope (SEM) using energy dispersive spectroscopy (EDS) to qualitatively identify composition of the various components.

\section{Results and Discussion}

The heavily cold worked microstructure of Region 1 (Fig. 4) suggests the inner brass tube was drawn to shape from tube stock. The presence of elongated inclusion stringers (Figure 6) embedded in the annealed brass grains 
of Region 2 (Figure 4) is also evidence of a prior drawing process for the outer tube.

To establish the proper inner diameter, the tubes may have undergone a process known as "balling" or "balling out," wherein a ball of the desired final tube diameter is forced through each tube. This processing step may have given rise to an interesting feature of the tube microstructure, evident in Fig. 7: The grains along the inner edge (top of the image) are

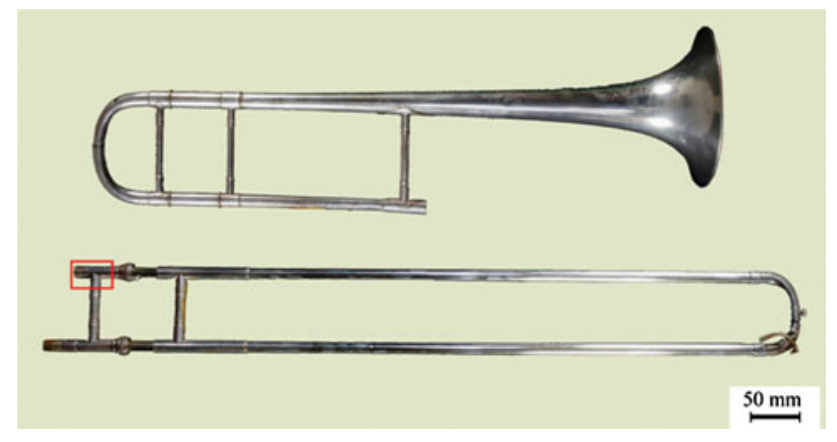

Fig. 1 Trombone components-the region of interest is boxed

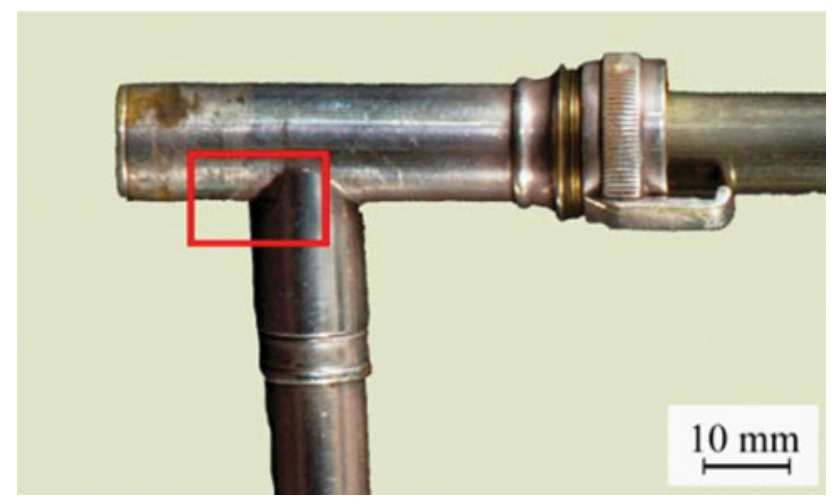

Fig. 2 Brace joint - the region sectioned and polished is boxed

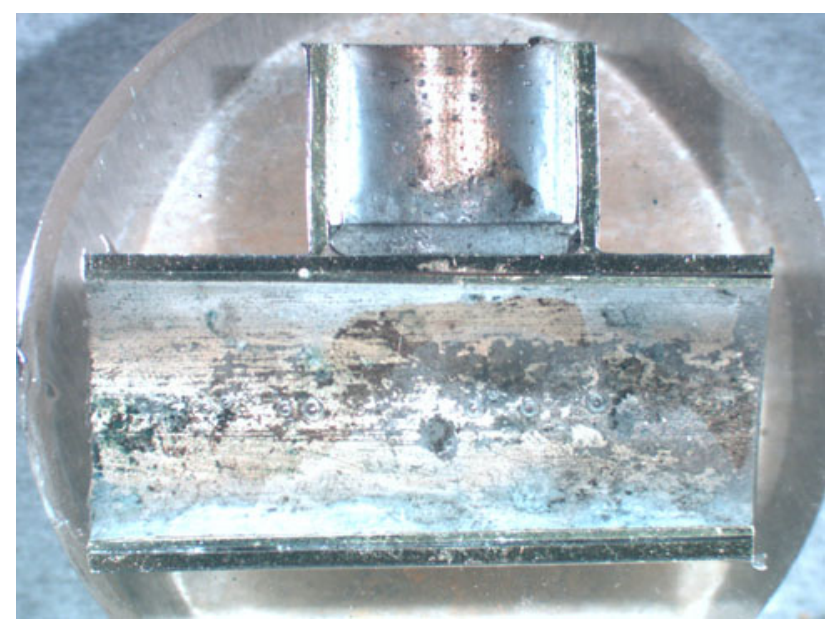

Fig. 3 Metallographic mount prepared from sample cut from location shown in Fig. 2 much smaller than the grains in the center of the sample. During the balling process, the grains at the inner tube surface will undergo more deformation than the grains in the center of the tube. These inner grains will therefore require less thermal energy to recrystallize: Recrystallization temperature will be lower for the severely cold-worked surface than for the remainder of the tube wall. The severe deformation will cause a large number of small grains to nucleate; these will grow rapidly to a stress-free, equiaxed configuration in the region of heavily cold-worked microstructure.

The brass tubes of Regions 3 and 5 are strictly parts of the joint, while the inner brass tube of Region 1 runs along the length of the trombone, forming the inner tube of the slide mechanism. The inner tube was not annealed after deformation, as evidenced by the microstructure, to maintain the high stiffness required for the slide. Other regions were annealed to ease fabrication of the joint. Results of

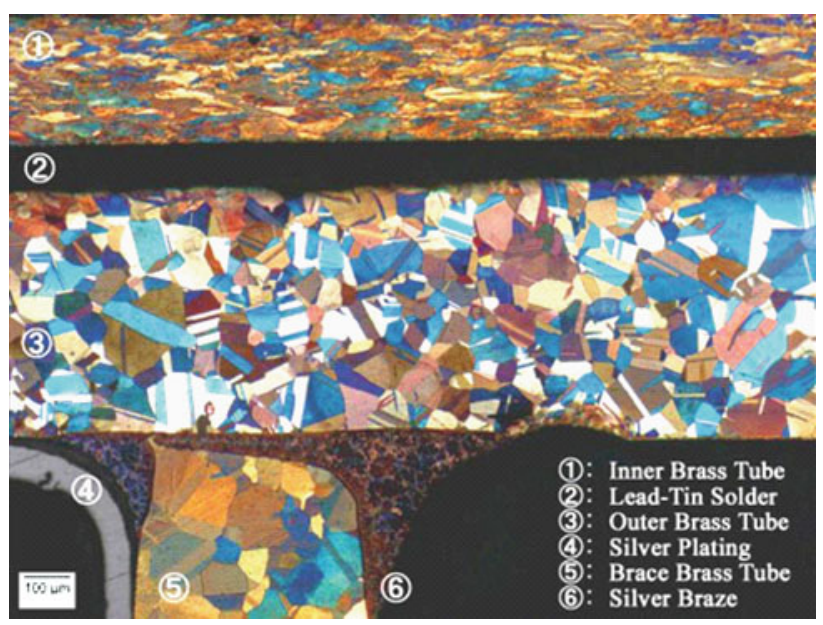

Fig. 4 Microstructure of the brace joint, etched with potassium dichromate, viewed with polarized light

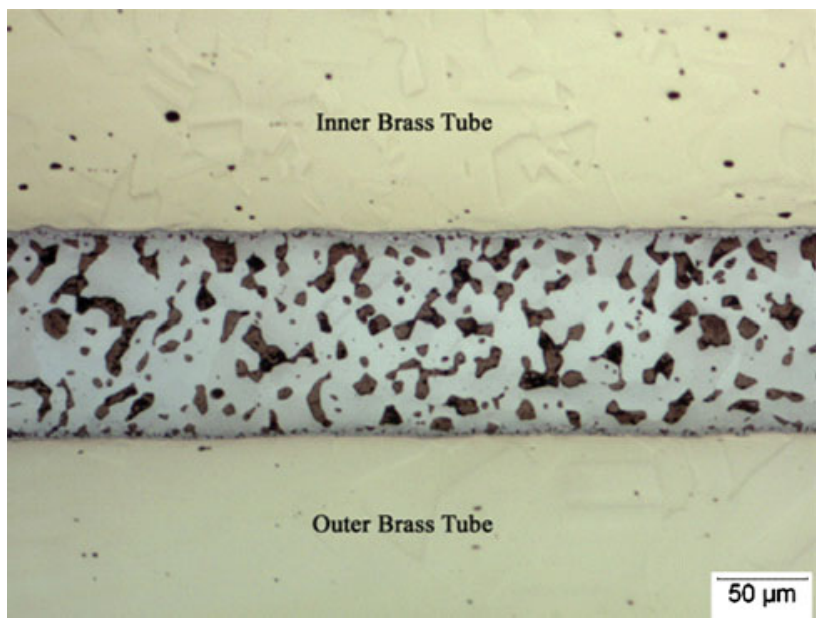

Fig. 5 Microstructure of the lead-tin solder, as polished (Region 2 in Fig. 4) 


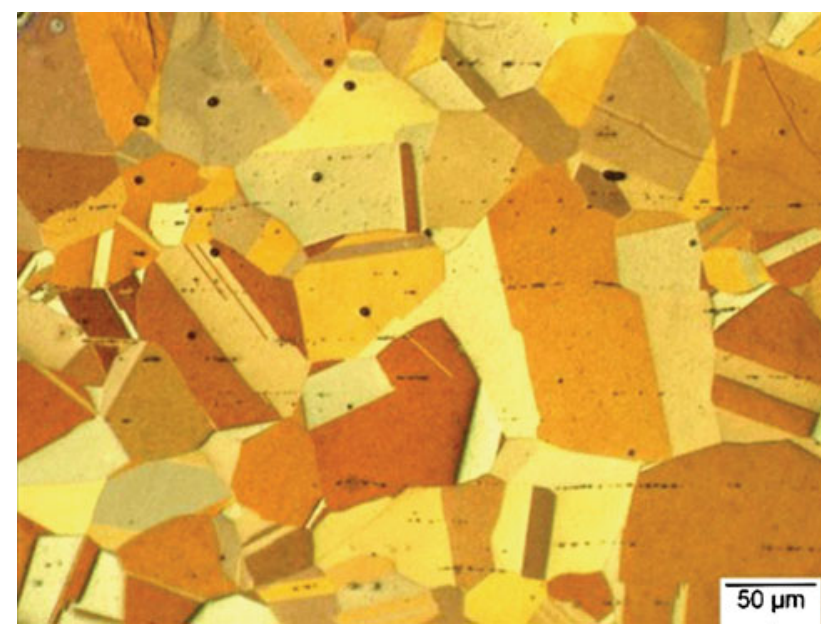

Fig. 6 Parallel lines of inclusion strings in the outer brass tube (Region 3 in Fig. 4), etched with potassium dichromate, viewed under partially crossed polars. The elongated strings embedded in annealed grains indicate the tube was first drawn then annealed

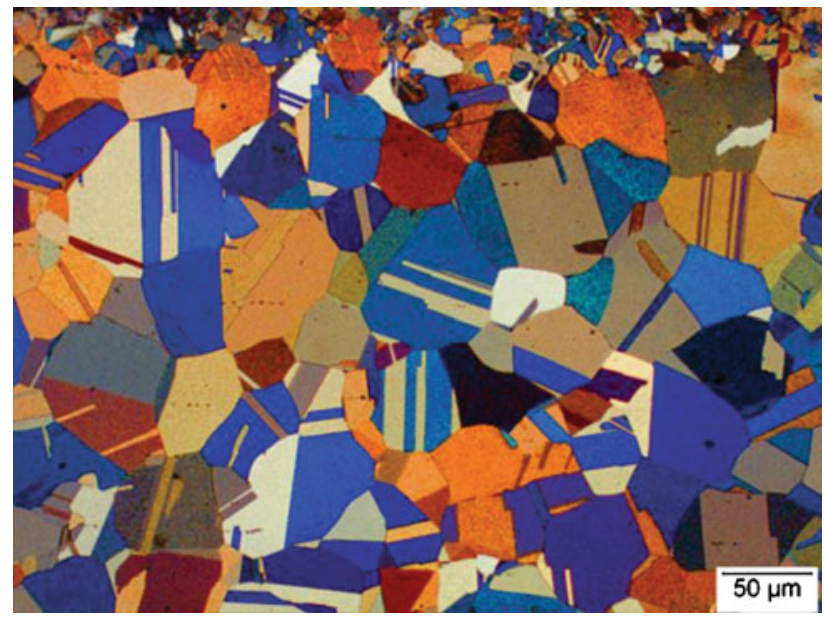

Fig. 7 Balled inside surface of the outer brass tube (Region 3 in Fig. 4), etched with potassium dichromate, viewed under polarized light. Note equiaxed grains formed after recrystallization of the heavily worked surface. The smaller grains (top) indicate the "balling" process indirectly decreased the final grain size

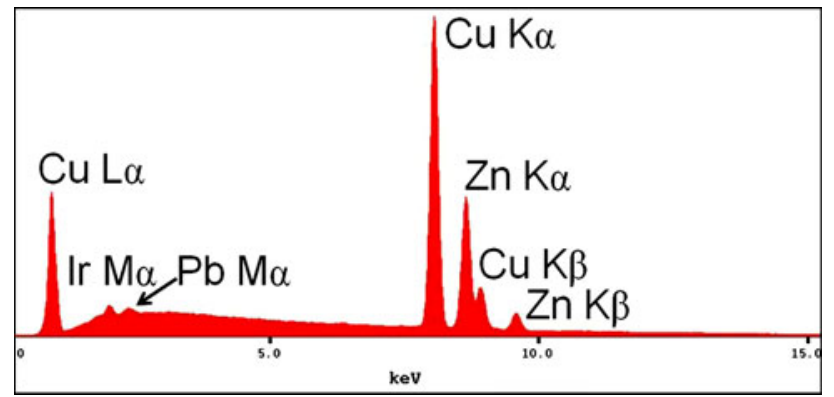

Fig. 8 EDS results of the outer and brace tubes shown in Fig. 4. The alloy is a leaded brass

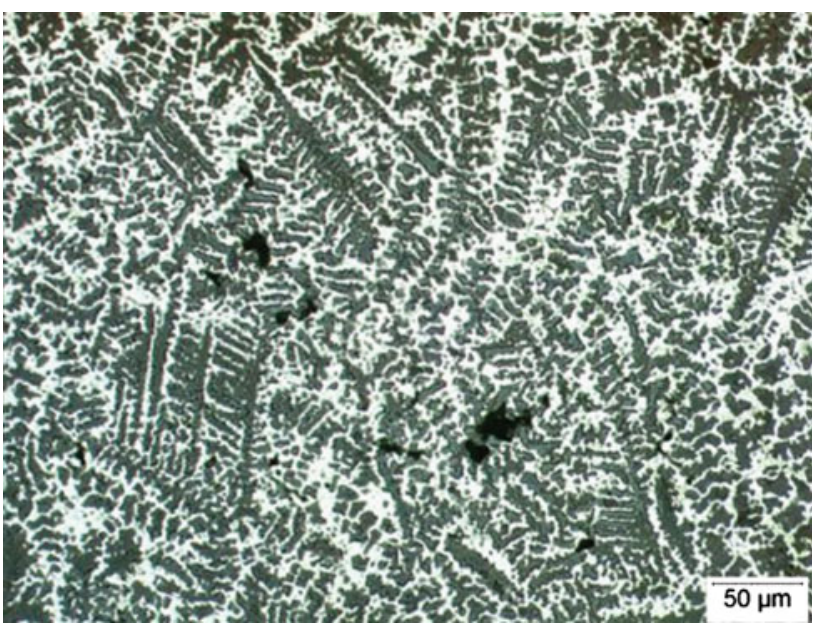

Fig. 9 Microstructure of the etched silver braze depicting $\mathrm{Ag}$ dendrites in a $\mathrm{Cu}-\mathrm{Zn}$ matrix (Region 6 in Fig. 4). Some areas with interdendritic porosity are also evident

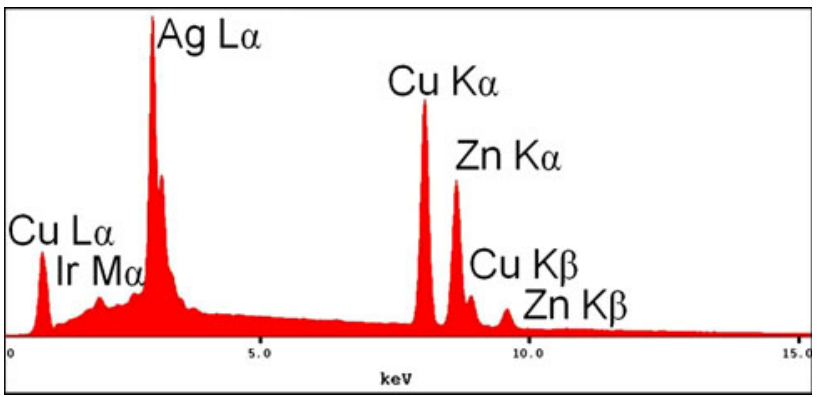

Fig. 10 EDS results of silver braze revealing constituents of $\mathrm{Ag}, \mathrm{Cu}$, and $\mathrm{Zn}$. Ir peak is due to Ir coating used during SEM sample preparation

EDS analysis of the inner and brace tubes indicate that a leaded brass was used for these components (Fig. 8). It is hypothesized that this alloy was chosen over other brasses for its increased ductility.

To construct the joint, the brace tube was brazed to the outer tube (Figs. 4, 9) to provide structural rigidity to the instrument. A silver-braze, composed of silver, copper, and zinc (Fig. 10), was selected for its improved wetting properties and relatively high hardness.

To attach the outer brass tube to the slide, thereby finishing the joint, the tubes were soldered using a lead-tin solder, as shown in Fig. 5. The lead-tin solder was probably selected for its low working temperature and low cost.

EDS analysis of the outer tube surface (Region 4 in Fig. 4) revealed that the trombone was electroplated with silver. Silver influences the sound quality of the instrument and gives it a "brightness" quality, as opposed to the darker tones generated by similar un-plated brass instruments.

Acknowledgement The authors would like to thank Samuel Lawrence for his help throughout this project. 